\title{
Haste does not always make waste: Expertise, direction of attention, and speed versus accuracy in performing sensorimotor skills
}

\author{
SIAN L. BEILOCK \\ Miami University, Oxford, Ohio \\ BENNETT I. BERTENTHAL \\ University of Chicago, Chicago, Illinois \\ and \\ ANNETTE M. MCCOY and THOMAS H. CARR \\ Michigan State University, East Lansing, Michigan
}

\begin{abstract}
In two experiments, we examined the attentional mechanisms governing sensorimotor skill execution across levels of expertise. In Experiment 1, novice and expert golfers took a series of putts under dual-task conditions designed to distract attention from putting and under skill-focused conditions that prompted attention to step-by-step performance. Novices performed better under skill-focused than under dual-task conditions. Experts showed the opposite pattern. In Experiment 2, novice and expert golfers putted under instructions that emphasized either putting accuracy or speed - the latter intended to reduce the time available to monitor and explicitly adjust execution parameters. Novices putted better under accuracy instructions. Experts were more accurate under speed instructions. In agreement with theories of skill acquisition and automaticity, novice performance is enhanced by conditions that allow for on-line attentional monitoring (e.g., skill-focused or accuracy instructions) in comparison with conditions that prevent explicit attentional control of skill execution (e.g., dual-task or speed constraints). In contrast, the proceduralized skill of experts benefits from environments that limit, rather than encourage, attention to execution.
\end{abstract}

Theories of skill acquisition and automaticity suggest that novice performance is supported by unintegrated task control structures held in working memory and attended in step-by-step fashion. Expert performance is thought to occur more automatically, largely controlled by procedures that run outside of working memory during execution (Anderson, 1993; Beilock \& Carr, 2001; Fitts \& Posner, 1967; Keele \& Summers, 1976). These differences in attentional demands of performance readily lead to predictions concerning how situations that alter the deployment of cognitive resources-either by drawing attention toward execution or by taking attention away_will affect performance across skill levels.

In two experiments, we manipulated deployment of attentional resources during on-line golf putting in an attempt to shed light on differences in control structures governing novice and expert sensorimotor skill execu-

The authors thank John Bell, golf teaching professional, for his consultation on this project and his suggestion that expert golf putting accuracy may be enhanced by performance time constraints, and Allen R. McConnell for his title suggestion. Correspondence concerning this article should be addressed to S. L. Beilock, Department of Psychology, Miami University, 202 Benton Hall, Oxford, OH 45056-1601 (e-mail: beilocsl@muohio.edu). tion. In Experiment 1, novice and expert golfers performed putts under skill-focused conditions intended to direct attention toward a component process of performance and also under dual-task conditions designed to draw attention away from execution via secondary task demands. If novices dedicate attention to controlling real-time execution, performance should be worse under dual-task than under skill-focused conditions, since nontask-related stimuli in the dual-task condition should occupy resources needed for primary skill execution (Nissen \& Bullemer, 1987). Once a skill becomes well learned, however, attention should not be needed for step-by-step execution. Experts, then, may not be negatively affected by secondary task constraints (Allport, Antonis, \& Reynolds, 1972). Yet attention prompted toward a component process of well-learned performance may disrupt automated processes that normally run as uninterrupted routines.

In Experiment 1, we found differences in the effect of attention as a function of skill level. Novice putting was more accurate under skill-focused than under dual-task attention conditions, but experts showed the opposite pattern. In Experiment 2, we pursued an implication of this conclusion - that any environment whose characteristics work to alter the attentional resources available for on-line execution may have different effects on performance as 
a function of expertise. For example, because attention takes time to deploy (Posner \& Snyder, 1975; Shiffrin \& Schneider, 1977), time constraints on performance may harm the execution of poorly learned skills by reducing the ability of novices to monitor and adjust task parameters, in relation to performance environments where time constraints are not an issue. However, the same temporal demands may help well-practiced execution if they limit attention to task control and guidance.

The notion that well-learned skills benefit from limited performance time flies in the face of the well-established speed-accuracy tradeoff (Fitts, 1954; Woodworth, 1899). Many studies have shown that the more rapidly a skill is performed, the less accurate it becomes. However, although this principle has been repeatedly demonstrated across both cognitive and motor skill domains (see, e.g., MacKay, 1982), it was discovered, and has most often been studied, in individuals possessing little previous exposure to the tasks they were performing. If control structures that govern performance differ as a function of skill level, it is possible that the speed-accuracy tradeoff does not generalize to well-learned skills. For this reason, in Experiment 2 we followed up the manipulation of direction of attention in Experiment 1 with an examination of the impact of time constraints on novice and expert putting performance.

\section{EXPERIMENT 1}

Recently, Beilock, Carr, MacMahon, and Starkes (2002) examined expert golfers' putting performance under a skill-focused condition that drew attention toward a component process of performance (i.e., monitoring the club swing and the cessation of the follow through) and under a dual-task condition in which individuals putted while simultaneously performing a secondary, auditory tone-monitoring task. In agreement with the framework described above, experienced golfers' putting performance was more accurate in the dual-task than in the skillfocused condition.

Experiment 1 replicated Beilock et al.'s (2002) findings with experienced golfers while extending the paradigm to novices. If, as theories of skill acquisition and automaticity predict, the control structures governing performance differ as a function of skill, then the impact of dual-task and skill-focused attention should have essentially opposite effects on novice and expert performance. Experts should be adversely affected by conditions that draw attention to proceduralized performance processes, in comparison with conditions that serve to limit such attentional control. Yet novices, who require declarative knowledge structures to be held in working memory and attended during performance, should benefit from performancedirected attention, as compared with situations that limit it. Because golf putting requires the assembly of many semi-independent processes, it may be a good skill in which to explore possible skill-level differences in the impact of manipulations that alter on-line attentional resources. Table 1 lists the component processes involved in a typical putt.

\section{Method}

\section{Participants}

The participants were undergraduate students. The novices $(n=18)$ had no previous golf experience. The experts $(n=18)$ had at least 2 years of high school varsity golf experience or a PGA handicap $<8$.

Table 1

Components Involved in a Typical Golf Putt, Separated Into Assessment and Execution Steps

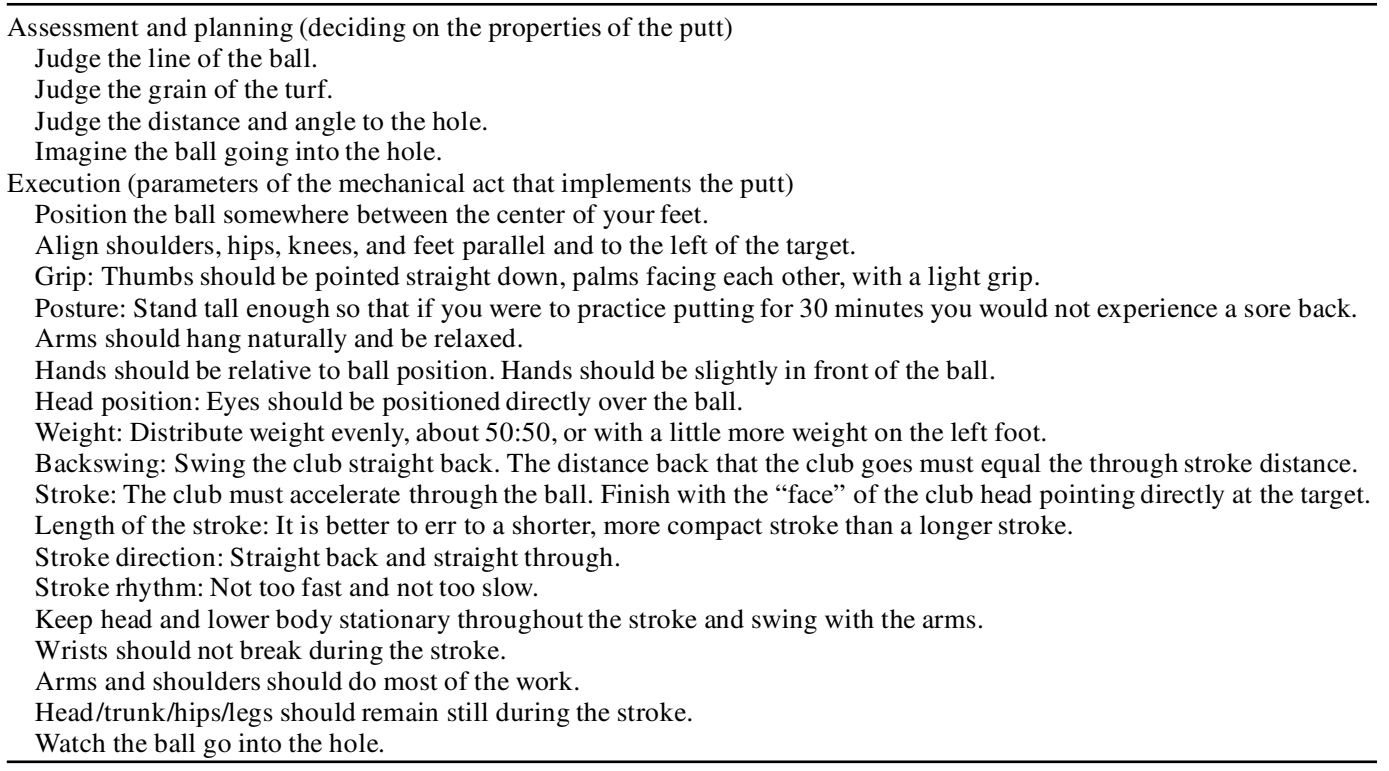




\section{Procedure}

The putting task was performed on a carpeted indoor green $(3 \times$ $3.7 \mathrm{~m}$ ) with a standard putter and ball. The individuals putted a golf ball as accurately as possible to a target, marked by a square of red tape, on which the ball was supposed to stop. The individuals putted from nine different spots, with three spots at three distances (120, 140 , and $150 \mathrm{~cm}$ ) from the target. All the participants followed the same random alternation of putting from the nine locations. A light next to each spot was illuminated prior to each putt to indicate the next putt's starting location.

The individuals performed 35 initial putts to familiarize them with our altered putting task, which required individuals to land the ball on a target rather than in a hole. The participants then took 20 practice putts in a single-task environment, 20 putts in a dual-task attention condition, and 20 putts in a skill-focused attention condition. The order of the two attention conditions was counterbalanced across participants.

Skill-focused condition. The individuals were instructed to monitor their swing and attempt to keep their club head straight as it traveled toward the target during their swing and follow-through. The participants were informed that in order to ensure that they were attending to the motion of the swing during the putt, they should say the word straight out loud at ball contact. This component of the swing was chosen as the basis for the skill-focused manipulation because a straight club head is thought to be an important component of a successful putt (Jones, Davis, Crenshaw, Behar, \& Davis, 1998).

Dual-task condition. The individuals putted while simultaneously listening to a series of tape recorded tones. The participants were instructed to monitor the tones, and each time they heard a specified target tone, they were to say the word tone out loud. The 500-msec target tone was played three times prior to the dual-task condition to familiarize the participants with this tone. The tones occurred randomly, once within every 2 -sec interval. The target tone occurred randomly, averaging once every four tones. The random placement of the tones within the 2-sec intervals and the random embedding of the target tone within the filler tones was designed to prevent anticipation of secondary task tone presentation.

\section{Results}

Putting accuracy was measured by the distance (in centimeters) between the center of the target and the ball stopped after each putt. The mean distance from the target of the 20 putts in the skill-focused and dual-task conditions was used as the measure of that condition's putting performance.

A 2 (expert, novice) $\times 2$ (skill focused, dual task) analysis of variance (ANOVA) on putting accuracy revealed a main effect of expertise $\left[F(1,34)=20.09, M S_{\mathrm{e}}=64.74\right.$, $p<.01]$, no main effect of condition $[F(1,34)=2.15$, $M S_{\mathrm{e}}=7.09$, n.s.], and a significant expertise $\times$ condition interaction $\left[F(1,34)=46.80, M S_{\mathrm{e}}=7.09, p<.01\right]$. A follow-up analysis showed this outcome to hold regardless of putting distance. There was no expertise $\times$ condition $\times$ distance interaction $\left[F(2,68)=1.08, M S_{\mathrm{e}}=17.02\right.$, n.s. $]$.

The experienced golfers were more accurate putters than were novices across the skill-focused and dual-task conditions (Figure 1). Furthermore, whereas the novices performed worse in the dual-task condition than in the skill-focused condition $[t(17)=3.20, p<.01]$, the experts' performance improved $[t(17)=7.65, p<.01]$.

Our main goal was to examine differences in performance across skill-focused and dual-task conditions as a

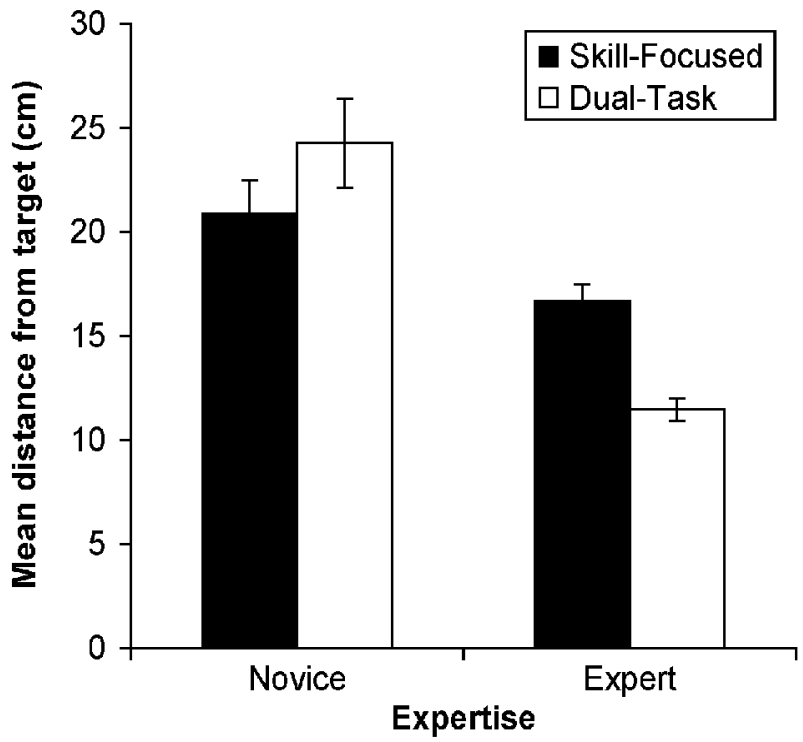

Figure 1. Mean distance (in centimeters) from the center of the target to where the ball stopped after each putt for the skillfocused and dual-task conditions for the novice and expert golfers in Experiment 1. Error bars represent standard errors of the mean.

function of expertise. However, comparisons of singletask practice performance with the attention conditions were also conducted. Novice dual-task putting was less accurate than was single-task practice $[M=21.31 \mathrm{~cm}$, $S D=5.55 \mathrm{~cm} ; t(17)=2.12, p<.05]$, whereas putting in the skill-focused condition was more accurate than it was in single-task practice $[t(17)=0.46$, n.s.], although not significantly. The experts showed the opposite pattern: Putting in the skill-focused condition was less accurate than it was in single-task practice $[M=12.10 \mathrm{~cm}, S D=$ $1.62 \mathrm{~cm} ; t(17)=6.13, p<.01]$, whereas performance in the dual-task condition was slightly more accurate than it was in single-task practice $[t(17)=0.98$, n.s. $]$.

\section{Secondary Task Performance}

Skill-focused condition. There were no instances in which individuals failed to say straight at ball contact across both groups of participants.

Dual-task condition. Failure to identify target tones occurred infrequently for both novices $(M=.33, S D=$ $.84)$ and experts $(M=.06, S D=.24)$ across all 20 putts in the dual-task condition. An analysis of errors across the dual-task condition was not interpretable because of error infrequency.

\section{Discussion}

The skill-focused and dual-task conditions had opposite effects on novice and experienced golf putting. Novice performance is thought to be based on declarative knowledge explicitly monitored on line. Thus, skillfocused attention drawn toward performance aids execution, in comparison with dual-task conditions that take 
such attention away. In contrast, expert performance is believed to be controlled by procedures operating largely unattended during real-time execution (Anderson, 1993; Fitts \& Posner, 1967; Keele \& Summers, 1976). Consequently, skill-focused attention disrupts performance, whereas dual-task conditions do not. The present results replicate Beilock et al.'s (2002) findings with experienced golfers. Furthermore, Experiment 1 extends this work to novices by highlighting differences in the impact of skillfocused and dual-task manipulations across skill levels.

\section{EXPERIMENT 2}

In Experiment 2, we further explored the attentional mechanisms driving sensorimotor skill execution through the examination of the impact of time constraints on novice and expert putting performance. It has been demonstrated across many different domains that controlled attentional processes take time to execute (Posner \& Snyder, 1975; Shiffrin \& Schneider, 1977). From a skill acquisition and automaticity framework, temporal demands may interfere with novice performance that is explicitly monitored in real time, especially if these demands prevent novices from attending to and adjusting performance parameters. However, time constraints may help skilled execution if they prevent performers from paying too much attention to task control and guidance-attention shown in Experiment 1 to be detrimental to expert performance.

\section{Method}

\section{Participants}

The participants were novice $(n=20)$ and experienced $(n=20)$ golfers, selected by utilizing the same skill-level criteria as those employed in Experiment 1.

\section{Procedure}

The participants performed a golf putting task similar to that in Experiment 1, with the exception that the individuals were instructed to putt to illuminated circular targets, created by a light projection from an LCD projector hung from the ceiling, rather than toward a target tape.

The participants first took 20 initial putts to a target, $5 \mathrm{~cm}$ in diameter, located $137 \mathrm{~cm}$ away. The order of the remaining putting blocks was counterbalanced across participants. The individuals took six blocks of 20 putts under accuracy instructions and six blocks of 20 putts under speed instructions (240 putts, total). As in Experiment 1, our main comparison of interest concerned differences in novice and expert putting performances across the instruction conditions. A single-task condition was not utilized in Experiment 2 because (1) it was not crucial to our main comparison and (2) single-task instructions that normally emphasize accuracy would have been similar to and hence redundant with the already included accuracy condition of Experiment 2.

The six blocks of putts under each instruction condition consisted of putts to targets of two different distances and three different sizes in a 2 (distance: 116 or $158 \mathrm{~cm}$ ) $\times 3$ (diameter: $9.14,12.45$, or $13.97 \mathrm{~cm}$ ) design. The target distances were chosen as representative of short putts taken in real-world golf. The target sizes were $15 \%$ smaller, $15 \%$ larger, and $30 \%$ larger, respectively, than a regulation PGA hole ( $10.80 \mathrm{~cm}$ in diameter), which were chosen to create variability in performance demands along a dimension that might affect the perceived difficulty of the task and might also increase the salience of the speed versus accuracy instructions.

Prior to the first putt of each block and again every fifth putt within a block, the participants were reminded of the instructions for that particular block. In the accuracy condition, the participants were instructed to try to land the ball on the target, taking as much time as they needed. In the speed condition, the participants were instructed to land the ball on the target. However, they were also instructed to execute their putts as quickly as possible, and they were informed that they had a maximum of $3 \mathrm{sec}$ to complete each putt (from the time the experimenter said "go" until the putter contacted the ball). The experimenter said "go" as soon as the participants took their putting stance, in order to prevent the individuals in the speed condition from preparing to take the putt before timing began.

All putts were timed on a stopwatch to the nearest 10th of a second. The timing of putts under accuracy instructions was initiated when the participants took their putting stance and stopped when the putter contacted the ball. The timing of putts under speed instructions was initiated when the experimenter said the start cue "go" (triggered by the participants' assuming their putting stance) and stopped when the putter contacted the ball. Following putting, the participants filled out a questionnaire designed to assess their perceptions of the impact of the speed and accuracy instructions on performance.

\section{Results}

Accuracy was measured by the distance (in centimeters) between the center of the target and where the ball stopped after each putt. This continuous measure was designed to provide maximum sensitivity in examining the effects of the target size, distance, and instructions on performance. A separate measure of target hits (defined as the ball's stopping anywhere on the target) was also recorded. This dichotomous measure was consistent with the instructions actually given to the participants. Both measures are relevant to real-world golf, in which the goal is to putt the ball anywhere in the hole (hits), but if one misses, to leave the ball as close to the hole as possible (accuracy).

\section{Target Size and Distance}

We began by assessing differences in putting performance and sensitivity to target distance and size as a function of expertise. A 2 (expert, novice) $\times 2$ (target distance: 116 or $158 \mathrm{~cm}) \times 3$ (target size: $9.14,12.45$, or $13.97 \mathrm{~cm})$ ANOVA on putting accuracy revealed a main effect of expertise $\left[F(1,38)=43.24, M S_{\mathrm{e}}=65.79, p<\right.$ $.01]$, an expertise $\times$ target distance interaction $[F(1,38)=$ $\left.11.96, M S_{\mathrm{e}}=6.99, p<.01\right]$, and an expertise $\times$ target size interaction $\left[F(2,76)=4.74, M S_{\mathrm{e}}=4.66, p<.05\right]$.

The experts were more accurate putters than were the novices across all target sizes and distances (see Table 2). The novices' putting accuracy improved from the smallest $(9.14 \mathrm{~cm})$ to the largest $(13.97 \mathrm{~cm})$ target $[t(19)=$ $2.91, p<.01]$, whereas the experts' putting accuracy did not differ as a function of target size $[t(19)=1.40$, n.s. $]$. In terms of target distance, both the experts and the novices putted more accurately to the closer target $[t(19)=$ $14.00, p<.01$, and $t(19)=14.28, p<.01$, respectively]. However, as with the impact of target size on putting accuracy, target distance affected the novices' putting accuracy more so than it did the experts' accuracy. This pat- 
Table 2

Mean Distance (in Centimeters) From the Center of the Target to Where the Ball Stopped After Each Putt (Accuracy) and Mean Percentage of Target Hits (\% Hits) for the Two Target Distances $(116$ and $158 \mathrm{~cm})$ and Three Target Sizes $(9.14,12.45$, and $13.97 \mathrm{~cm}$ ) for the Novice and Expert Golfers in Experiment 2

\begin{tabular}{|c|c|c|c|c|c|c|}
\hline \multirow[b]{3}{*}{ Group } & \multicolumn{6}{|c|}{ Target Size } \\
\hline & \multicolumn{2}{|c|}{$9.14 \mathrm{~cm}$} & \multicolumn{2}{|c|}{$12.45 \mathrm{~cm}$} & \multicolumn{2}{|c|}{$13.97 \mathrm{~cm}$} \\
\hline & $M$ & $S E$ & $M$ & $S E$ & $M$ & $S E$ \\
\hline \multicolumn{7}{|c|}{ 116-cm Target Distance } \\
\hline Novice & & & & & & \\
\hline Accuracy & 15.36 & 0.80 & 14.82 & 0.66 & 14.24 & 0.85 \\
\hline$\%$ Hits & 18.31 & 1.30 & 23.70 & 1.88 & 29.94 & 2.61 \\
\hline \multicolumn{7}{|l|}{ Expert } \\
\hline Accuracy & 8.76 & 0.63 & 8.79 & 0.56 & 9.75 & 0.62 \\
\hline$\%$ Hits & 25.53 & 3.05 & 31.30 & 2.75 & 39.42 & 2.47 \\
\hline \multicolumn{7}{|c|}{ 158-cm Target Distance } \\
\hline \multicolumn{7}{|l|}{ Novice } \\
\hline Accuracy & 24.03 & 1.44 & 22.35 & 1.19 & 21.82 & 0.96 \\
\hline$\%$ Hits & 1.50 & 0.49 & 3.76 & 0.69 & 5.38 & 0.84 \\
\hline \multicolumn{7}{|l|}{ Expert } \\
\hline Accuracy & 14.79 & 0.92 & 14.53 & 0.75 & 14.67 & 0.86 \\
\hline$\%$ Hits & 4.13 & 0.82 & 9.54 & 1.15 & 15.75 & 2.08 \\
\hline
\end{tabular}

tern of smaller effects of size and distance on the experts' performance is not surprising, considering that expert golfers must often adapt to performing in diverse environmental conditions (e.g., the angle of the hole or the lie of the green) to ensure optimal skill execution in realworld situations.

A similar ANOVA on target hits revealed main effects of expertise $\left[F(1,38)=19.89, M S_{\mathrm{e}}=0.02, p<.01\right]$, target distance $\left[F(1,38)=279.93, M S_{\mathrm{e}}=0.01, p<.01\right]$, and size $\left[F(2,76)=41.28, M S_{\mathrm{e}}=0.005, p<.01\right]$. Regardless of target size or distance, the experts had more target hits than did the novices, and for both skill levels, target hits increased as target size increased and target distance decreased. As can be seen in Table 2, putting accuracy and target hits followed a similar pattern, with the exception that the less sensitive dichotomous measure of target hits revealed main effects of target size and distance, rather than interactions with expertise, as was obtained in the putting accuracy analyses. Thus, we succeeded in creating a situation in which putting performance varied with situational constraints and golf expertise. We now turn to our main hypothesis concerning the impact of temporal demands on novice and expert performance.

\section{Speed Versus Accuracy Instructions}

Putting performance. Much as in Experiment 1, a 2 (expert, novice) $\times 2$ (accuracy instruction, speed instruction) ANOVA on putting accuracy revealed a main effect of expertise $\left[F(1,38)=43.31, M S_{\mathrm{e}}=21.90, p<\right.$ $.01]$, no main effect of instruction $[F(1,38)=2.81$, $M S_{\mathrm{e}}=4.93$, n.s.], and an expertise $\times$ instruction interaction $\left[F(1,38)=25.85, M S_{\mathrm{e}}=4.93, p<.01\right]$. As is shown in Figure 2, the novices performed better when putting under accuracy instructions than under speed instructions $[t(19)=3.85, p<.01]$. By contrast, the ex- perts performed better under speed than under accuracy instructions $[t(19)=3.57, p<.01]$.

A similar ANOVA on target hits produced a main effect of expertise $\left[F(1,38)=19.87, M S_{\mathrm{e}}=0.005, p<\right.$ $.01]$, no main effect of instruction $(F<1)$, and an expertise $\times$ instruction interaction $[F(1,38)=20.89$, $\left.M S_{\mathrm{e}}=0.002, p<.01\right]$. The novices had more hits under accuracy instructions $[t(19)=2.78, p<.05]$. The experts had more hits under speed instructions $[t(19)=$ $3.71, p<.01$; Figure 2]. Thus, speed versus accuracy instructions exerted opposite influences on the novices'
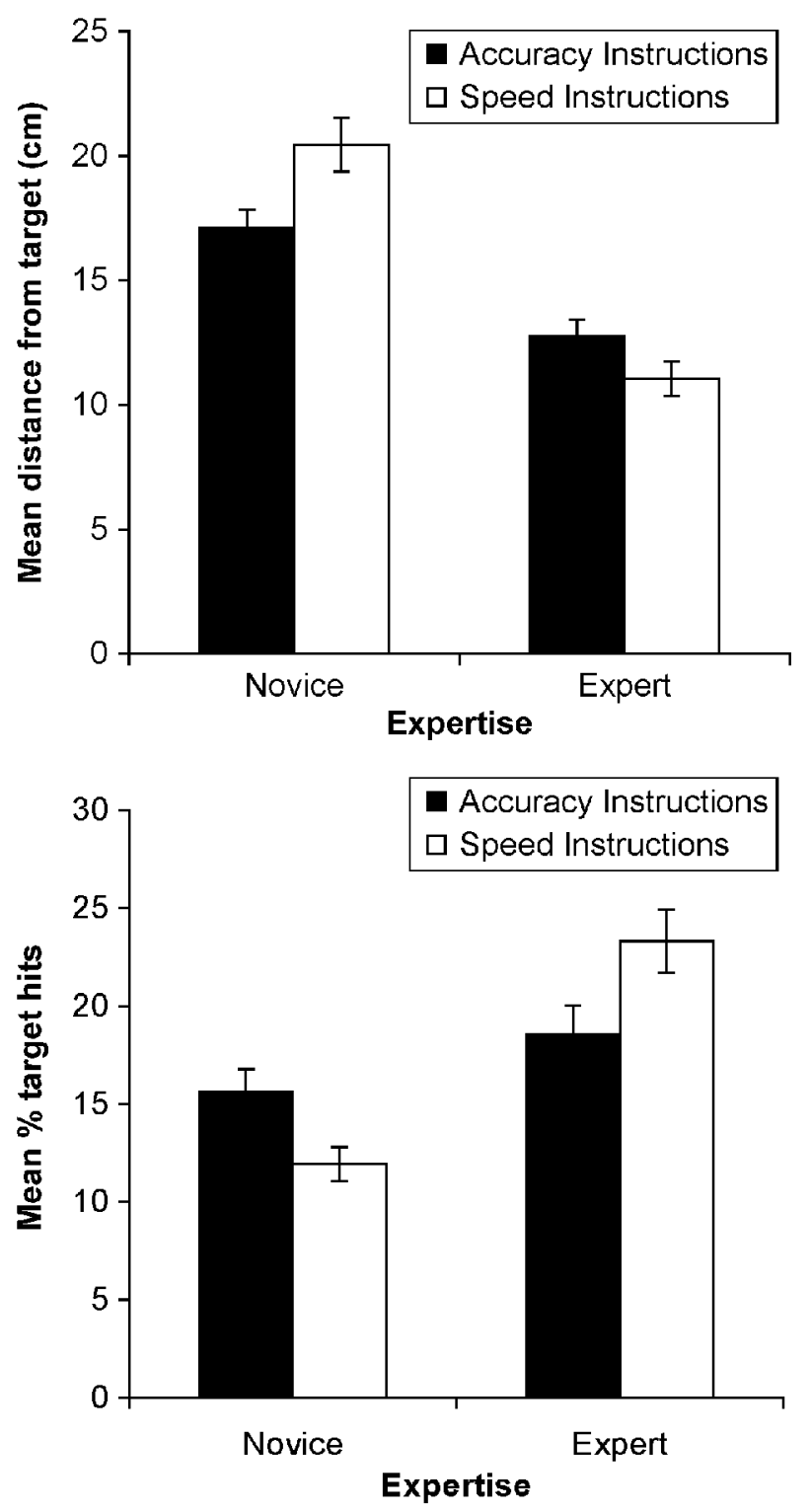

Figure 2. Mean distance (in centimeters) from the center of the target to where the ball stopped after each putt (upper panel) and mean percentage of target hits (lower panel) for the accuracy instructions and speed instructions for the novice and expert golfers in Experiment 2. Error bars represent standard errors of the mean. 
and the experts' performance, as measured by both putting accuracy and target hits. Although the novices performed better under accuracy than under speed instructions, the experts performed better under speed than under accuracy instructions.

Putting time. Putting time was analyzed to ensure that the individuals performed faster when speed was stressed. In the speed condition, three individual putts (two expert, one novice putt; $0.06 \%$ of all speed putts) took over the 3 -sec maximum. As in the accuracy and hits analyses, these putts were retained in the present analysis to obtain the most accurate picture of the relationships among instructions, putting performance, and putting time. Excluding these putts would not have altered the results.

A 2 (expert, novice) $\times 2$ (accuracy instruction, speed instruction) ANOVA on putting time revealed a main effect of instruction $\left[F(1,38)=329.01, M S_{\mathrm{e}}=0.133, p<\right.$ $.01]$, a main effect of expertise $\left[F(1,38)=4.59, M S_{\mathrm{e}}=\right.$ $0.44, p<.05]$, and no expertise $\times$ instruction interaction $(F<1)$. The novices and the experts alike took longer to putt under accuracy instructions (novices, $M=2.50 \mathrm{sec}$, $S D=.80 \mathrm{sec}$; experts, $M=2.87 \mathrm{sec}, S D=.53 \mathrm{sec})$ than under speed instructions (novices, $M=1.07 \mathrm{sec}, S D=$ $.32 \mathrm{sec}$; experts, $M=1.34 \mathrm{sec}, S D=.36 \mathrm{sec}$ ). In addition, however, the experts took longer than did the novices to putt, regardless of instructions. The experts' longer performance time is consistent with work in domains such as physics problem solving, which demonstrates that experts often spend more time assessing and analyzing problems than do novices (Chi, Feltovitch, \& Glaser, 1981). However, because the experts took longer to putt across conditions, it is possible that putting time, rather than expertise or instruction, led to the differential impact of instructions on expert and novice putting performance, reported above.

To examine this notion, we conducted a 2 (expert, novice) $\times 2$ (accuracy instruction, speed instruction) ANOVA on each putt's accuracy divided by the time taken to perform that putt. This analysis of accuracy per unit time produced the same results as those above, which suggests that instructions altered the efficiency or effectiveness with which time was used, and not simply total time per se. Furthermore, correlations between each participant's putting time and accuracy revealed a negative mean correlation among novices $(M=-.07$, $S D=.12$ ) but a positive mean correlation among experts $(M=.10, S D=.10)$. These mean correlations, though small, were significantly different from each other $[t(38)=4.74, p<.01]$ and from $0[t(19)=2.48, p<$ .03 , and $t(19)=4.43, p<.01$, respectively]. Putting accuracy in the present study was an error score. Thus, the longer the novices took to putt, the better they performed. Conversely, the longer the experts took to putt, the worse they performed. This is consistent with the accuracy per unit time and the putting performance analyses, reported above.

Questionnaire. The participants reported on a 7-point scale how they felt the accuracy and speed instructions affected performance. The scales ranged from 1 (negatively affected performance) to 7 (positively affected performance), with 4 (didn't affect performance) as the midpoint. A 2 (expert, novice) $\times 2$ (accuracy question, speed question) ANOVA revealed a marginally significant expertise $\times$ question interaction $\left[F(1,38)=3.04, M S_{\mathrm{e}}=\right.$ $21.8, p<.09]$. The novices reported speed instructions $(M=3.10, S D=1.37)$ as having a significantly more negative impact on performance than accuracy instructions $\operatorname{did}[M=4.00, S D=1.20 ; t(19)=2.27, p<.04]$. In contrast, the experts reported speed instructions $(M=$ $3.95, S D=1.43$ ) as having a slightly less negative impact on performance than accuracy instructions did $[M=3.70, S D=1.56 ; t(19)=0.47]$, although this difference was not significant.

Thus, the participants' rated perceptions of the instructions tended to follow their objectively measured impact: The novices perceived the speed constraints as detrimental to performance in comparison with accuracy instructions, whereas the experts showed a trend toward the opposite perception. Participant comments on the questionnaire reinforce this finding. One novice stated that the speed instructions "made me rush and not think enough about the task," whereas one expert reported that the speed instructions aided performance because they "didn't let me go into the depth of the actual putt." Another expert stated, "The faster I did, the better I did."

\section{Discussion}

Novices perform worse under instructions to putt as quickly as possible relative to instructions that do not limit execution time. The opposite is true for experts. Expert performance, at least in the type of sensorimotor task we have studied, is enhanced by speed constraints, in comparison with performance when time pressure is not an issue. Novices need time to attend to and control performance. The proceduralized performances of experts do not require, and appear to be adversely affected by, unlimited execution time-perhaps because that time affords them the counterproductive opportunity to explicitly attend to and monitor automated execution processes (Beilock et al., 2002).

In this study, total time to perform a putt (including both preparation and movement time) was used as the time parameter of interest. Although early speed-accuracy tradeoff work (e.g., Fitts, 1954; Woodworth, 1899) addressed only movement time, this phenomenon has since been expanded to skills with both preparation and movement time components (e.g., forced-choice discriminations, spatial judgments, and spoken sentence reproduction; see MacKay, 1982). Whether speed instructions in the present study impacted putting preparation time, movement time, or both is an issue for future research.

\section{GENERAL DISCUSSION}

In two experiments, we examined the on-line attentional demands of novice and expert sensorimotor skill execution. The results demonstrated that whereas novice 
performance benefits from enhanced attention to execution in comparison with conditions that take such attention away, expert skill execution excels in situations that limit, rather than encourage, attention to execution. These findings highlight differences in the attentional control structures governing performance across skill levels and complement a growing body of literature that suggests that well-learned performances may falter under conditions that provide the opportunity for the reinstantiation of explicit skill-monitoring and control mechanisms that are needed by novices but should be exercised with caution by experts. The detrimental effects of enhanced attention to skilled performance can be seen not only in complex skills such as golf putting, but in more basic skills we take for granted. For example, Wulf and colleagues (Wulf \& Prinz, 2001) have suggested that directing performers' attention to their movements through internal focus feedback on a dynamic balance task interferes with the automated control processes that normally regulate balance movements outside of conscious scrutiny. Thus, for real-time execution by experts, there may be truth in the Nike motto "Just do it."

\section{REFERENCES}

Allport, D. A., Antonis, B., \& Reynolds, P. (1972). On the division of attention: A disproof of the single channel hypothesis. Quarterly Journal of Experimental Psychology, 24, 225-235.

ANDERSON, J. R. (1993). Rules of mind. Hillsdale, NJ: Erlbaum.

BEILOCK, S. L., \& CARR, T. H. (2001). On the fragility of skilled performance: What governs choking under pressure? Journal of Experimental Psychology: General, 130, 701-725.

Beilock, S. L., CARR, T. H., MAcMahon, C., \& Starkes, J. L. (2002).
When paying attention becomes counterproductive: Impact of divided versus skill-focused attention on novice and experienced performance of sensorimotor skills. Journal of Experimental Psychology: Applied, 8, 6-16.

Chi, M. T., Feltovitch,P. J., \& Glaser, R. (1981). Categorization and representation of physics problems by experts and novices. Cognitive Science, 5, 121-152.

FITTS, P. M. (1954). The information capacity of the human motor system in controlling the amplitude of movement. Journal of Experimental Psychology, 47, 381-391.

FitTs, P. M., \& Posner, M. I. (1967). Human performance. Belmont, CA: Brooks/Cole.

Jones, B. T., Davis, M., Crenshaw, B., Behar, T., \& Davis, M. (1998). Classic instruction in golf. New York: Broadway.

KeEle, S. W., \& Summers, J. J. (1976). The structure of motor programs. In G. E. Stelmach (Ed.), Motor control: Issues and trends (pp. 109-142). New York: Academic Press.

MACKAY, D. G. (1982). The problems of flexibility, fluency, and speedaccuracy trade-off in skilled behavior. Psychological Review, 89, 483-506.

Nissen, M. J., \& Bullemer, P. (1987). Attentional requirements of learning: Evidence from performance measures. Cognitive Psychology, 19, 1-32.

PosNer, M. I., \& SNYDER, C. R. R. (1975). Attention and cognitive control. In R. L. Solso (Ed.), Information processing and cognition: The Loyola Symposium (pp. 55-85). Hillsdale, NJ: Erlbaum.

ShIFFrIN, R. M., \& SCHNEIDER, W. (1977). Controlled and automatic human information processing: II. Perceptual learning, automatic attending, and a general theory. Psychological Review, 84, 127-190.

WoODWORTH, R. S. (1899). The accuracy of voluntary movement. Psychological Review Monograph Supplements, 3(2, Whole No. 13).

WUlF, G., \& PRINZ, W. (2001). Directing attention to movement effects enhances learning: A review. Psychonomic Bulletin \& Review, 8, 648-660.

(Manuscript received July 8, 2002;

revision accepted for publication November 7, 2002.) 